\title{
Ground-based Vision Cloud Image Classification based on Extreme Learning Machine
}

\author{
Zhengping $\mathrm{Wu}^{1}$, Xian $\mathrm{Xu}^{2}$, Min Xia, ${ }^{3, *}$, Meifang $\mathrm{Ma}^{4}$ and $\mathrm{Lin}^{2}{ }^{3}$ \\ ${ }^{1}$ Institute of Engineering Education, Sanjiang University, Nanjing 210012 China; ${ }^{2}$ School of Electronics and \\ Information Engineering, Sanjiang University, Nanjing 210012 China; ${ }^{3}$ Jiangsu Collaborative Innovation Center on \\ Atmospheric Environment and Equipment Technology, Nanjing University of Information Science and Technology, \\ Nanjing 210044, China; ${ }^{4}$ Blackrock Microsystems, Salt Lake City, USA
}

\begin{abstract}
Cloud radiation properties and distribution significantly affect the forecasting accuracy, climate monitoring effectiveness and global climate's change. A simple method was proposed to automatically recognize four different sky conditions (cirrus, cumulus, stratus and clear sky) by means of extracting some features from visual images that can be used for training classifier. In this paper, texture features, color features and SIFT features were extracted and extreme learning machine was used for cloud-type classification under different experimental conditions. The experiment results show that the proposed approach using texture features, color features and SIFT features together showed better performance than using these features alone or any two of them together. The accurate identification rate of cirrus, cumulus, stratus and clear sky were $87.67 \%, 90.75 \%, 74.50 \%$ and $93.63 \%$, respectively with an average of $86.64 \%$. Under the same experimental condition, the proposed method outperformed the artificial neutral network (ANN), k-nearest neighbor (KNN) and support vector machine (SVM).
\end{abstract}

Keywords: Cloud classification, Texture features, Color features, Sift features, Extreme learning machine.

\section{INTRODUCTION}

Clouds are important part of the earth's heat balance and hydrological cycle, as the clouds' change determines the earth's radiation balance and interaction with solar and at the same time plays an important role in the global climatic change $[1,2]$. As it is known that different cloud-types with different shapes, sizes, and physical structure reflect features of weather through changes in the atmosphere. However, the net effect of clouds is still not clear and will cause major uncertainties in climate models and climate predictions [3]. Recently, the observations of the amount of cloud, cloud form and height of cloud base are based on the visual judgment of the meteorological observers and satellite remote sensing. Human observations, however, will bring high costs so that the development of automatic devices to detect and quantify cloud amount and type has become an inexorable trend [4].

Satellite cloud images can provide a wide range of large scale distribution of the cloud structure information, but for thin clouds and low clouds, they are limited by spatial resolution and unknown surface effects. The range of ground-based observation is small, which can provide local distribution

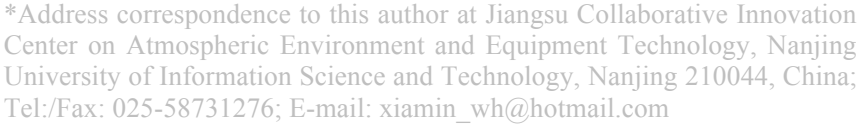
Tel:/Fax: 025-58731276; E-mail: xiamin_wh@hotmail.com information such as clouds size, arrangement and cloud height distribution. In recent years, a lot of ground-based sky imaging instruments have been developed, FOR the improvement of both the hardware [e.g., charge-coupled devices (CCDs) and digital image processing (DIP) techniques. These instruments include whole sky imager (WSI) [5], total sky imager (TSI) [6], whole sky infrared cloud measuring system (WSIRCMS) [7], and ground-based total-sky cloud Imager (TCI) [8]. At present, research of the ground-based total-sky cloud classification mainly concentrated in the visible cloud images. Buch et al. [9] using WSI data, presented a binary decision tree model to distinguish five sky types: cirrus, cumulus, stratus, altocumulus and clear sky based on the analysis of texture features, brightness information and location information. Peura et al. [10] extracted features like cloud edge sharpness, boundary shape, different degree of fibrous and edge information from all-sky cloud images, dividing the cloud into ten types, using $\mathrm{K}$-means clustering with total accuracy rate of $65 \%$. Singh and Glennen [11] presented an approach of cloud classification for common digital images for extracting numerous features from the grayscaled images, using K-nearest neighbor and neural network method to distinguish five different sky conditions, but the authors acknowledged their results as uncertain. Calbo and Sabburg [12] used some possible criteria for whole sky-images to classify eight predefined sky conditions, which include statistical features, features based on Fourier transform, and features that need prior distinction between clear and cloudy 
pixels. But this method only achieved an accuracy of $62 \%$. Heinle et al. [13] used K-nearest neighbor method for classification based on the spectral features and gray level co-occurrence matrix texture features. Kazantzidis et al. [14] based on Heinle's work, not only used texture and statistical color features, but also considered the solar zenith angle, the cloud coverage, the visible fraction of solar disk and the existence of raindrops in seven kinds of sky.

This paper presents some global features and local features extracted from digital images of the sky and which can be useful for cloud-type classification, based on recent cloud classification research status at home and abroad. In section 2, the texture features, color features and SIFT features extracted from cloud images are presented. In section 3 , the clouds classifier-extreme learning machine is introduced. Under different experimental conditions, the performance and results of the algorithm are discussed in section 4. Finally, section 5 summarizes the conclusions of this research and suggests possible future investigations on cloud-type identification from ground-based sky images.

\section{FEATURES FOR CLOUD-TYPE RECOGNITION}

\subsection{Texture Features}

Texture features reflect the visual features of images through the pixels and distribution regularity of its surrounding grayscale space. They describe the local characteristics of the image, according to the local mode number, type and their relationship with texture texton to describe the texture. Texture analysis mainly has four methods [15], including statistical analysis method, structural analysis method, model based analysis and signal analysis method. This paper used statistical analysis approach, adopting two texture features: gray-level cooccurrence matrix (GLCM) proposed by Haralick et al. [16] and visual perception texture features proposed by Tamura $e t$ al. [17] .

\subsubsection{Gray-level Co-occurrence Matrix}

Gray-level co-occurrence matrix reflects the joint probability occurrence of gray levels $i$ and $j$ for two pixels with a defined spatial relationship in an image. Element[i,j] of the matrix is defined by calculating the probability of a pixel with value $i$ being adjacent to a pixel with pixel $j$. The probability called $\mathrm{P}(\mathrm{i}, \mathrm{j}, \mathrm{d}, \theta)$ is defined as:

$\mathrm{P}(\mathrm{i}, \mathrm{j}, \delta, \theta)=\left\{(x, y) \mid \begin{array}{c}z(x, y)=i, z(x+D x, y+D y) \\ =j ; x, y=0,1,2, \cdots, N-1\end{array}\right\}$

Here, $\delta=(\mathrm{Dx}, \mathrm{Dy})$ and the direction $\theta$ is always set as: $0^{\circ}, 45^{\circ}, 90^{\circ}, 135^{\circ}$. Also, six features are used namely, energy, contrast, entropy, homogeneity, correlation and moment of inertia.

\subsubsection{Tamura's Texture Features}

Tamura put forward six basic texture features based on human subjective psychological measurement: coarseness, contrast, directionality, line likeness, regularity and roughness. Generally, the first three components are especially important for image retrieval. Coarseness describes significantly spatial changes of grey levels, contrast measures the brightness of image, and directionality refers to the direction of the grey values in the image. In this paper, these three features were used as Tamura texture feature.

\subsection{Color Features}

Color feature is a global feature, which describes the surface properties of the object or scene included in image. Comparing with other features, color features have a high robustness because of the little dependence on size, direction and angle of the image itself. For the expression of color features two questions are considered: first, choosing the appropriate color space to describe the color features; secondly, the quantitative method will be used to transform color features expression into the form of vector. This article used color moment to express image color, which was proposed by Stricker and Orengo [18]. This method utilizes the concept of the moment in linear algebra for the distribution of colors in an image expressed at the moment. Due to the fact that color distribution information is concentrated in the lower order moments, therefore, only the first order moments (mean), the second order moments(variance) and the third order moment(skewness) were used to describe the color distribution. Unlike color histogram, color moment could also be used to describe image without quantitative image features.

\subsection{SIFT Descriptor}

In this work, the bag of words model was used to process sift descriptor. The basic idea of BOW is to regard images as orderless collection of independent local image block and has shown impressive levels of performance [19-21], and provides description for each image block [22]. By clustering the description, it obtains a dictionary which contains visual vocabulary (usually SIFT keypoints). A BOW is then built as histogram over visual word occurrences. According to the high dimension vector representation of training set, the classifier is generated which is used to classify the image.

Main steps of constructing a BOW descriptor:

1) Through the detection of image block, descriptors are generated. In this paper, dense sampling was used because research by Fei-Fei et al. [23] observed that dense features work better for some classification and that random sampling of key-points is more appropriate than selecting key points by detectors [24]. The overlapped grid is $16 \times 16$ pixels, with spacing of 8 pixels. Following this, Low's high dimensional SIFT descriptor was used to describe each $16 \times 16$ patche. Each descriptor consists of 128 dimensions, and these vectors represent local invariant point in the image.

2) Combining the feature point vector and K-means [25], clustering is then utilized to group similar image patches (SIFT descriptor format) into M bins, where $\mathrm{M}$ is the vocabulary size in the experiments. 
3) Counting the number of occurrences of each word in the image from dictionary, and thus the image can be represented as a k-dimensional histogram.

Therefore, histogram features were taken as the input of classifier for cloud classification.

\section{EXTREME LEARNING MACHINE}

In this work, extreme learning machine was used as classifier. As a kind of single hidden-layer feed-forward network, compared to the traditional methods, it can randomly select the number of hidden layer neurons in the network. The input weights and the hidden layer deviation can be randomly assigned and output layer weights can be calculated by the least squares method. ELM has fast learning speed with a higher generalization performance than the traditional gradient-based learning algorithms, and solves the common problem of learning epochs, learning rate, stopping criteria, and local minima [26].

Assuming that there are $\mathrm{N}$ distinct samples $\left(x_{i}, t_{i}\right)$, $x_{i}=\left[x_{i 1}, x_{i 2}, \cdots, x_{i n}\right]^{T} \in R^{n}, t_{i}=\left[t_{i 1}, t_{i 2}, \cdots, t_{i m}\right]^{T} \in R^{m}$. The standard SLFN with $\mathrm{N}^{\prime}$ hidden neurons and activation function $\mathrm{g}(x, y)$ are mathematically modeled as $\sum_{i=1}^{\mathrm{N} \prime} \beta_{i} g_{i}\left(x_{j}\right)=\sum_{i=1}^{\mathrm{N} \prime} \beta_{i} g\left(w_{i} \cdot x_{j}+b_{i}\right)=o_{j}$, where $w_{i}=$ $\left[w_{i 1}, w_{i 2}, \cdots, w_{i n}\right]^{T}$ is the weight vector connecting the ith hidden neurons and the input neurons, $\beta_{i}=\left[\beta_{i 1}, \beta_{i 2}, \cdots, \beta_{i m}\right]^{T}$ is the weight vector connecting hidden neurons and the output neurons, and $b_{i}$ is the threshold of the ith hidden neurons. $w_{i} \cdot x_{j}$ indicates the inner product of $w_{i}$ and $x_{j}$. As show in (Fig. 1), network structure diagram is characterized of three layers.

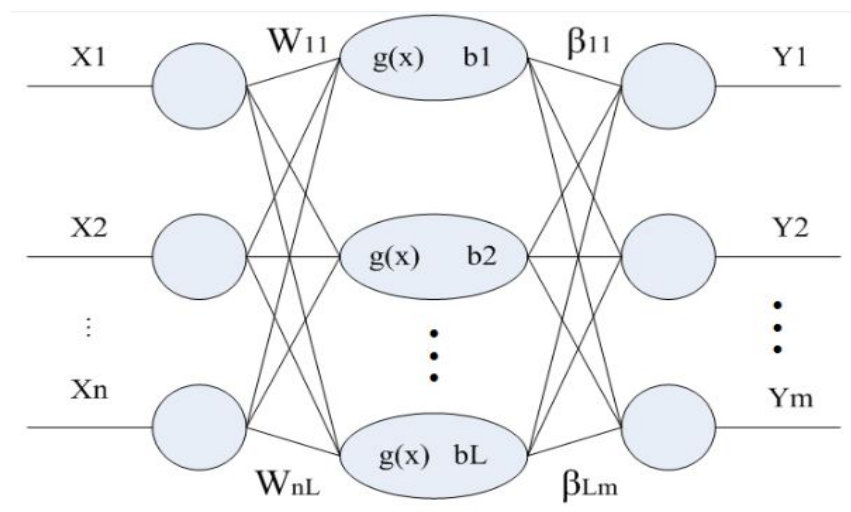

Fig. (1). The structure of ELM model.

That the standard SLFNs can approximate these $\mathrm{N}$ samples with zero error means that $\sum_{j=1}^{\mathrm{N}^{\prime}}\left\|o_{j}-t_{j}\right\|=0$. Thus, there also exist $w_{i}, \beta_{i}$ and $b_{i}$ such that

$\sum_{i=1}^{\mathrm{N} \prime} \beta_{i} g\left(w_{i} \cdot x_{j}+b_{i}\right)=t_{j} \quad j=1,2, \cdots, N$

According to the above $\mathrm{N}$ equations, following relation is obtained.

$\mathrm{H} \beta=\mathrm{T}$
Where $\mathrm{H}$ is the hidden-later output matrix. The ith column of $\mathrm{H}$ denotes the ith hidden neuron output with respect to inputs $x_{1}, x_{2}, \cdots, x_{N}$

$\mathrm{H}=\left[\begin{array}{ccc}g\left(w_{1} \cdot x_{1}+b_{1}\right) & \cdots & g\left(w_{\mathrm{N}^{\prime}} \cdot x_{1}+b_{\mathrm{N}^{\prime}}\right) \\ \vdots & \cdots & \vdots \\ g\left(w_{1} \cdot x_{N}+b_{1}\right) & \cdots & g\left(w_{\mathrm{N}^{\prime}} \cdot x_{N}+b_{\mathrm{N}^{\prime}}\right)\end{array}\right]_{N \times \mathrm{N}^{\prime}}$

$\beta=\left[\beta_{1}^{T}, \beta_{2}^{T}, \cdots, \beta_{\mathrm{N}^{\prime}}^{T}\right]_{m \times \mathrm{N}^{\prime}}^{T}$,

$\mathrm{T}=\left[t_{1}^{T}, t_{2}^{T}, \cdots, t_{N}^{T}\right]_{m \times \mathrm{N}}^{T}$

If the activation function $\mathrm{g}$ is infinitely differentiable, according to the theorem of extreme learning machine [27], the following equation is obtained:

$\|H \beta-T\|=0$

Therefore, training an SLFN is equivalent to finding least squares solutions $\hat{\beta}$ of the linear system $\mathrm{H} \beta=\mathrm{T}$, i.e. $\hat{\beta}=H^{\prime} T . H^{\prime}$ is the Moore-Penrose generalized inverse of matrix $\mathrm{H}$.

Based on above knowledge, the steps of algorithm are as follows:

1) The original data is used to train a neural network. $x_{i}=\left[x_{i 1}, x_{i 2}, \cdots, x_{i n}\right]^{T}$ is the training set, $\mathrm{g}(x, y)$ is the activation function and $\mathrm{N}^{\prime}$ is the hidden neurons. Randomly assigning input weight $w_{i}$ and bias $b_{i}$, to calculate output matrix $\mathrm{H}$ according to equation (4).

2) Calculating the output weight $\beta$.

3) Sending the testing data to ELM, four classification values can be obtained. The maximum value is selected as the final classification result.

4) Repeating steps 1-3 S times, computing the average of these $\mathrm{S}$ predicting values as the classification accuracy.

The proposed cloud-type identification research processes are shown as (Fig. 2).

\section{RESULTS AND DISCUSSION}

\subsection{Cloud Classification based on Texture Feature}

In this work, nine parameters of texture features were selected for cloud-type recognition, where the hidden neurons $\mathrm{N}^{\prime}$ of ELM were respectively selected as 12,15 , $20,25,30$, and 35 and the recognition rates for four skytypes were $63.93 \%, 65.23 \%, 67.63 \%, 67.45 \%, 72.02 \%$ and $71.76 \%$ respectively. When $\mathrm{N}^{\prime}=30$, a higher average recognition rate was observed. Table 1 shows the recognition rate and misjudgment rate of all kinds of sky-types when $\mathrm{N}^{\prime}=30$. From Table 1, the overall success rate was about $72.02 \%$, but only $61.63 \%$ correct classification rate was obtained for stratus, where $26.5 \%$ and $10.02 \%$ were mistaken recognition rate for cirrus and cumulus respectively. According to these results shown in Table 1, it was observed that the recognition rates only based on texture features could not achieve good performance. 


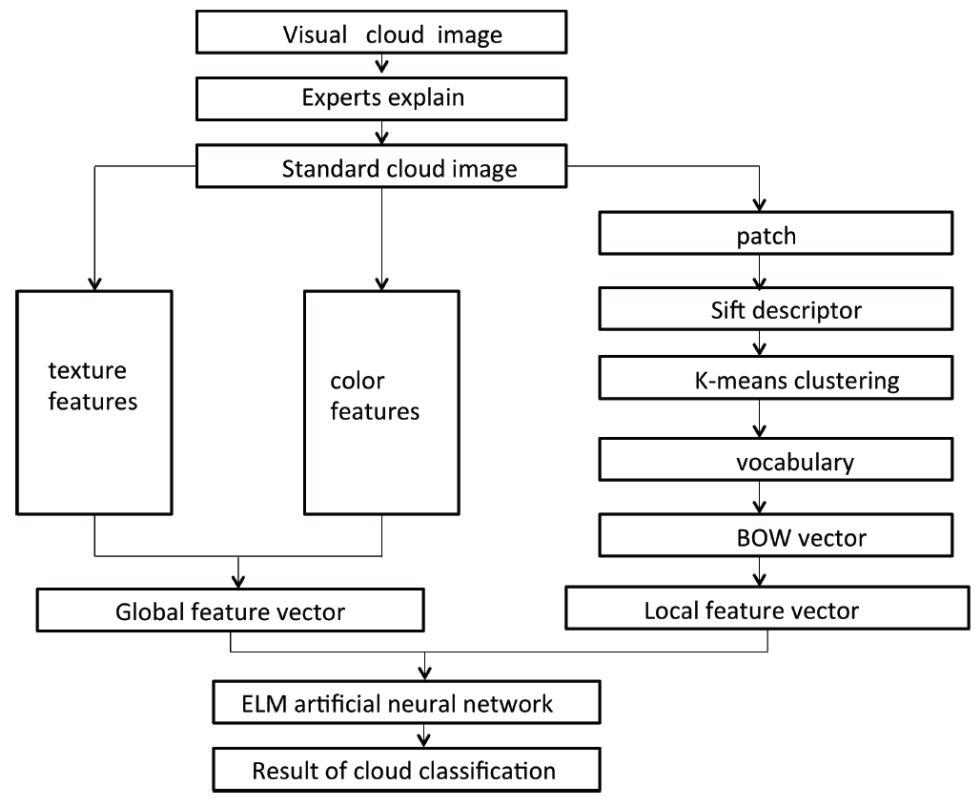

Fig. (2). The algorithm process of cloud recognition.

Table 1. Confusion matrix using texture features alone when $\mathbf{N}^{\prime}$ is set to 30 .

\begin{tabular}{|c|c|c|c|c|c|}
\hline & Cirrus & Clear sky & Cumulus & Stratus & 0.2650 \\
\hline \hline Cirrus & 0.7025 & 0.0020 & 0.3139 & 0.0185 & 0.1002 \\
\hline Clear sky & 0.0721 & 0.9619 & 0.0007 & 0.6163 & 0.7202 \\
\hline Cumulus & 0.1154 & 0.0186 & 0.6002 & 0.0852 & 0.0172 \\
\hline Stratus & 0.1100 & 0.02 & & \\
\hline
\end{tabular}

Table 2. Confusion matrix using color features alone when $\mathbf{N}^{\prime}$ is set to 12 .

\begin{tabular}{|c|c|c|c|c|c|}
\hline & Cirrus & Clear sky & Cumulus & Stratus & 0.0886 \\
\hline \hline Cirrus & 0.5769 & 0.0150 & 0.2269 & 0.0350 & 0.1459 \\
\hline Clear sky & 0.1259 & 0.9653 & 0.0031 & 0.7305 & 0.7103 \\
\hline Cumulus & 0.2354 & 0.0022 & 0.5683 & 0.2017 \\
\hline Stratus & 0.0617 & 0.0174 & & 0 \\
\hline
\end{tabular}

\subsection{Cloud Classification based on Color Features}

There were nine color features used to identify the cloud type, where the hidden neurons $\mathrm{N}^{\prime}$ of ELM were selected as $8,10,12,15,20,25$, and 30 , and the recognition rates for four sky-types were $6.21 \%, 69.61 \%, 71.03 \%, 70.29 \%$, $69.97 \%, 70.73 \%$ and $70.28 \%$ respectively. When $\mathrm{N}^{\prime}=12$, which shows a higher average recognition rate. Table 2 gives the recognition rates and misjudgment rates for four different sky-types when $\mathrm{N}^{\prime}=12$. Compared with texture features, the average accuracy was similar, but the recognition rates for cirrus and cumulus were only $57.69 \%$ and 56.83 respectively, while, $23.54 \%$ and $22.69 \%$ were mistaken as cumulus and cirrus, resulting in great confusion.

\subsection{Cloud Classification based on Sift Features}

In this paper, BOW model was used to process sift descriptors and the value of $\mathrm{M}$ for k-means was 500, as a result the histogram of bin $=500$ was obtained, and then normalized. Finally, 500 dimensional vector can be used to represent the image. When the hidden neurons N'of ELM were $1200,1500,1800,20,2500$, and 3000 , the recognition rates for four sky-types were $80.66 \%, 81.89 \%, 82.49 \%$, $82.95 \%, 83.82 \%$, and $83.49 \%$. When $\mathrm{N}^{\prime}=2500$, it reflected 
Table 3. Confusion matrix using sift features alone when $\mathbf{N}^{\prime}$ is set to 2500 .

\begin{tabular}{|c|c|c|c|c|c|}
\hline & Cirrus & Clear sky & Cumulus & Stratus & 0.0647 \\
\hline \hline Cirrus & 0.8311 & 0.0830 & 0.1088 & 0.0006 & 0.1087 \\
\hline Clear sky & 0.0535 & 0.8574 & 0.0005 & 0.8260 & 0.8382 \\
\hline Cumulus & 0.0474 & 0.0059 & 0.0526 & 0.0007 & \\
\hline Stratus & 0.0068 & 0.0007 & & \\
\hline
\end{tabular}

a higher average recognition rate. Table 3 shows the confusion matrix using sift features with $\mathrm{N}^{\prime}=2500$. Comparing the texture and color results, the overall performance was observed to be better, and the recognition rate for clear sky was slightly higher than the other three kinds of sky type.

\subsection{Cloud Classification based on Texture Features and Color Features}

For texture features and color features, 18 components were used for cloud type classification. When the hidden neurons $\mathrm{N}^{\prime}$ of ELM were selected as 12, 15, 20, 25, 30, 35, and 40, the recognition rates were $70.32 \%, 73.03 \%, 75.33 \%, 76.35 \%$, $76.81 \%, 71.23 \%$, and $71.12 \%$ respectively. As shown in Table 4 , the recognition rates for four cloud types using texture and color features were better than the one that only uses any one of them. The cloud was misjudged, however, because more than $20 \%$ of cumulus was mistaken for cirrus.

\subsection{Cloud Classification based on Color Features and Sift Features}

Color features and sift features included 509 components for classification. When the hidden neurons N'of ELM were $900,1200,1400,1500,1800,2000$, and 2500 the recognition rates were $82.59 \%, 83.59 \%, 84.63 \%, 84.91 \%, 85.59 \%$, $85.79 \%$, and $85.89 \%$ respectively.

When $\mathrm{N}^{\prime}=2500$, it showed a higher average recognition rate of $85.89 \%$. Table 5 shows the confusion matrix when $\mathrm{N}^{\prime}=2500$. The results illustrates that using two features together is better than using only color features or sift features. This showed that combining two features can be useful for improving the recognition rate.

\subsection{Cloud Classification based on Texture Features and Sift Features}

Based on 9 components of texture features and 500 components of sift features, when the hidden neurons N'of ELM were 900, 1200, 1500, 1800, 2000, 2500, and 3000, the

Table 4. Confusion matrix using texture features and color features when $\mathrm{N}^{\prime}$ is set to 30 .

\begin{tabular}{|c|c|c|c|c|c|}
\hline & Cirrus & Clear sky & Cumulus & Stratus & 0.1848 \\
\hline \hline Cirrus & 0.8177 & 0.0092 & 0.2420 & 0.0004 & 0.1308 \\
\hline Clear sky & 0.0001 & 0.9899 & 0.0007 & 0.6841 & 0.7681 \\
\hline Cumulus & 0.1607 & 0 & 0.5806 & 0.1767 & \\
\hline Stratus & 0.0215 & 0.0008 & & 0.609 \\
\hline
\end{tabular}

Table 5. Confusion matrix using color features and sift features when $\mathrm{N}^{\prime}$ is set to 2500 .

\begin{tabular}{|c|c|c|c|c|c|}
\hline & Cirrus & Clear sky & Cumulus & Stratus & 0.1357 \\
\hline \hline Cirrus & 0.8741 & 0.0344 & 0.0734 & 0.0304 & 0.0332 \\
\hline Clear sky & 0.0026 & 0.9499 & 0 & 0.8008 & 0.8589 \\
\hline Cumulus & 0.0955 & 0.0146 & 0.1157 & 0.0012 & \\
\hline Stratus & 0.0278 & 0.0109 & \\
\hline
\end{tabular}


Table 6. Confusion matrix using texture features and sift features when $\mathrm{N}^{\prime}$ is set to 2500 .

\begin{tabular}{|c|c|c|c|c|c|}
\hline & Cirrus & Clearsky & Cumulus & Stratus & 0.2361 \\
\hline \hline Cirrus & 0.9324 & 0.0332 & 0.0552 & 0.0010 & 0.2458 \\
\hline Clear sky & 0.0213 & 0.9355 & 0 & 0.5171 & 0.8241 \\
\hline Cumulus & 0.0254 & 0.0313 & 0.0116 & 0.0332 & \\
\hline Stratus & 0.0210 & 0 & & \\
\hline
\end{tabular}

recognition rates for four cloud types were $79.39 \%, 80.49 \%$, $81.10 \%, 81.58 \%, 81.76 \%, 82.41 \%, 82.16 \%$. From the results it was observed that the overall accuracy was better than using only texture features. The results of recognition rates and misjudgement rates for four different cloud types are shown in Table 6. From the confusion matrix, it can be seen that the recognition rate for cirrus, clear sky and cumulus was higher, but lower for stratus .

\subsection{Cloud Classification based on Texture Features, Color Features and Sift Features}

By combining texture features, color features and sift features, there were total 518 components. when the hidden neurons $\mathrm{N}^{\prime}$ of ELM were 1200, 1400, 1500, 1800, $2000,2500,3000$, the recognition rates for four cloud types were $85.69 \%, 86.02 \%, 85.59 \%, 86.22 \%, 86.64 \%, 86.33 \%$, $86.60 \%$, and the overall performance was better than the ones discussed above. The highest recognition rate was $86.64 \%$ with $\mathrm{N}^{\prime}=2000$. Table 7 shows the confusion matrix using these three features. Four kinds of sky type the recognition rate reached more than $70 \%$, and achieved good results. This illustrates that global features and local features combined together are more conducive to improve the recognition rate, and enhance the classification performance.

\subsection{Experimental Comparison and Misjudgment Analysis}

Since the support vector machine (SVM), k-nearest neighbor algorithm (KNN), and Back Propagation (BP) artificial neural network are popular methods for cloud classification, therefore, the proposed approach of this work is benchmarked against KNN, SVM and BP. Fig. (3) shows the classification results for KNN, SVM, BP and ELM. The proposed method has been proved to be more robust for cloud type classification.

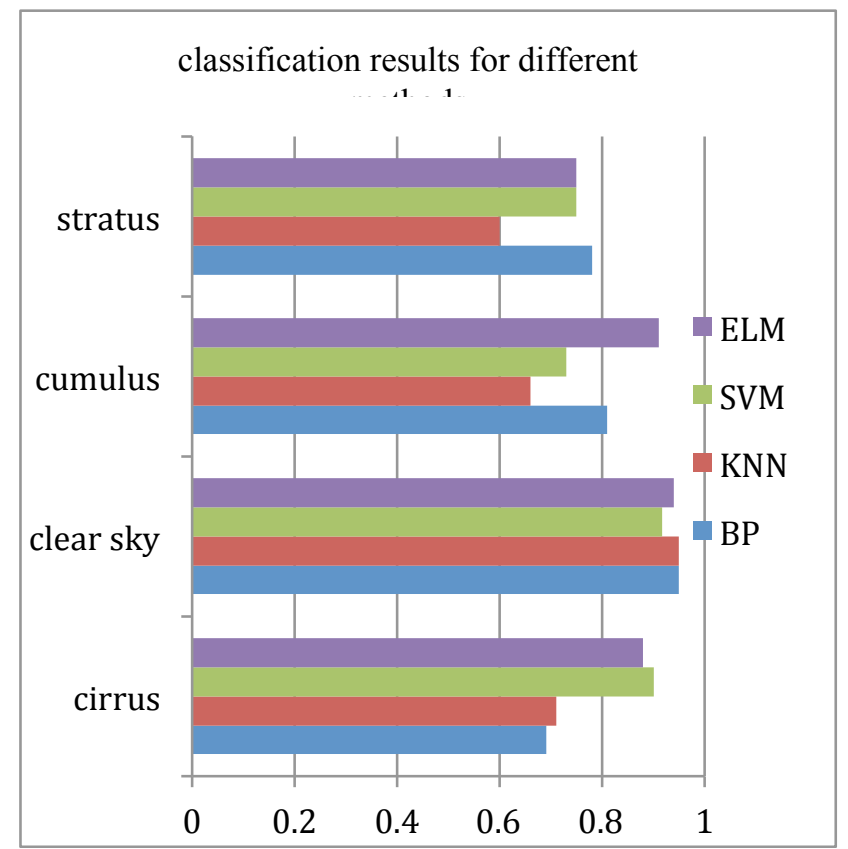

Fig. (3). Classification results for KNN, SVM, BP and ELM.

When using texture features, color features and sift features together for cloud classification, the average accuracy was observed to be $86.64 \%$, and the recognition rates for cirrus, clear sky, cumulus and stratus were $87.67 \%$, $93.63 \%, 90.75 \%$ and $74.5 \%$ respectively. In the classification, there was $9.46 \%$ cirrus mistakenly recognized as cumulus. Analyzing these $9.46 \%$ samples to find out that these mistaken samples were large, and contained small cumulus, known as complex sky, resulted in misjudgment. Fig. 4(a) shows the sample that cirrus mistook for cumulus. At the same time, $2.43 \%$ cirrus was mistaken as stratus, and these samples covered the sky, showing the layered

Table 7. Confusion matrix using texture features, color features and sift features when $\mathrm{N}^{\prime}$ is set to 2000.

\begin{tabular}{|c|c|c|c|c|c|}
\hline & Cirrus & Clear sky & Cumulus & Stratus & Mean \\
\hline \hline Cirrus & 0.8767 & 0.0375 & 0.0583 & 0.1061 & 0.0037 \\
\hline Clear sky & 0.0044 & 0.9363 & 0.0017 & 0.1452 & 0.7450 \\
\hline Cumulus & 0.0946 & 0.0209 & 0.9075 & 0.0326 & 0.8664 \\
\hline Stratus & 0.0243 & 0.0054 & & 0 \\
\hline
\end{tabular}


characteristics. Fig. 4(b) shows the sample that cirrus mistook for stratus.

For the classification of cumulus, there were $5.83 \%$ samples misjudged as cirrus, and it was found that these samples had common characteristic of fragmented body. There were $3.26 \%$ samples misjudged as stratus, and it was observed that the body of these samples covered the sky, showing the layered characteristics. Fig. (5) shows the cumulus sample mistaken for cirrus and stratus.

For the classification of stratus, there were $10.61 \%$ samples mistakenly recognized as cirrus. These cloud samples exhibited irregular fragments and some bits and pieces under the cloud. Fig. 6(a) shows the stratus sample mistaken for cirrus. Also, $14.52 \%$ samples were mistaken for cumulus, and analysis of these samples identified that the body of these cloud samples covered the sky, but there still remained a massive cloud body, showing the cumuliformis characteristics. Fig. 6(b) shows the stratus sample mistaken for cumulus. There were little samples of cumulus, stratus and cirrus misjudged as clear sky because of insignificant cloud covered in the image.

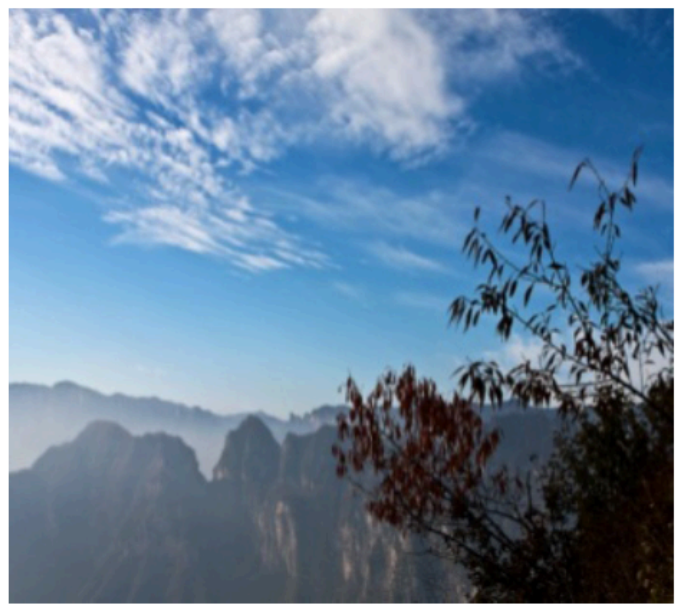

(a)

Fig. (4). Cirrus are mistaken for cumulus (a) and stratus (b).

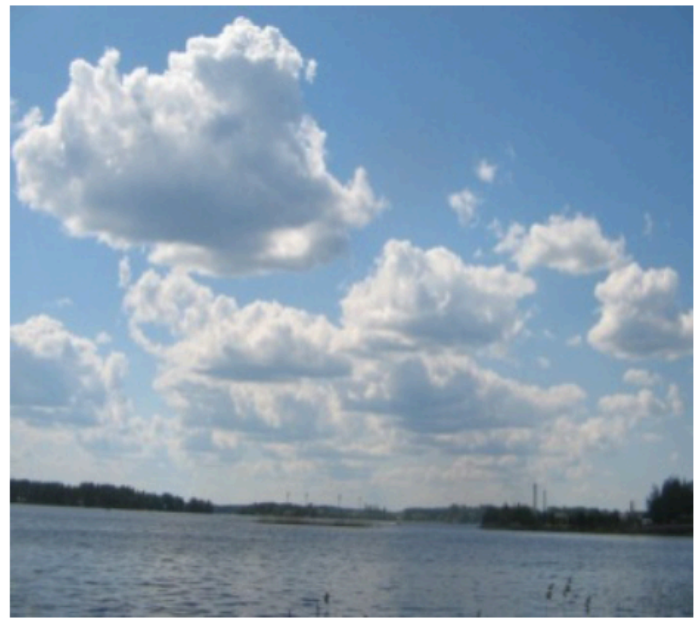

(a)

\section{CONCLUSION}

Cloud classification is an important part of automatic observation of sky, and accurate and quantitative automatic cloud observation is useful for numerous climatic models, hydrologic and atmospheric research. In this paper, a cloud classification method was proposed to automatically recognize different kinds of digital sky images. In the article, the global features of cloud image, i.e. texture features and color features are not discussed, but sift features were analyzed by means of BOW model. Finally, a detailed analysis of cloud image with three features and extreme learning machine was carried out. Experimental results demonstrate that combining these three features for classification is better than using only one feature or any two of them.

In nature, the sky often presents a wide series of different cloud type at the same time, such as cirrus and cumulus or cirrostratus and stratocumulus frequently appear together. In order to avoid misclassification caused by this phenomenon, it is suggested to divide the image into sub-images initially, and the blocks can be smaller. In addition, it is important to

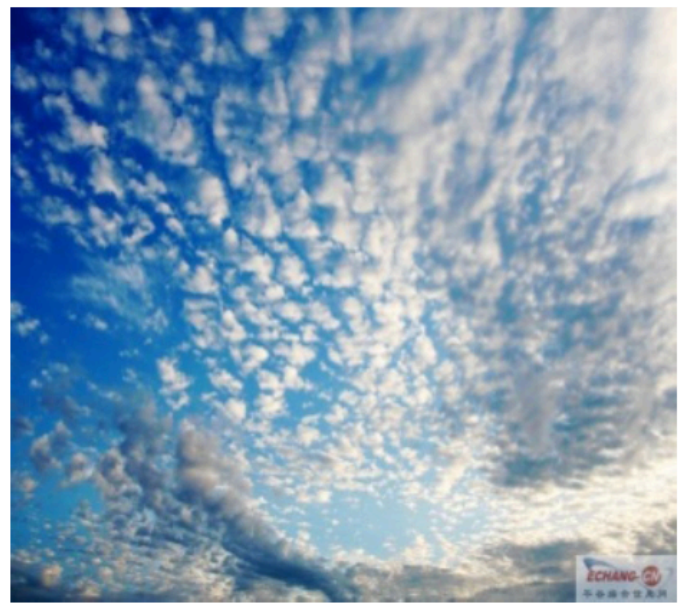

(b)

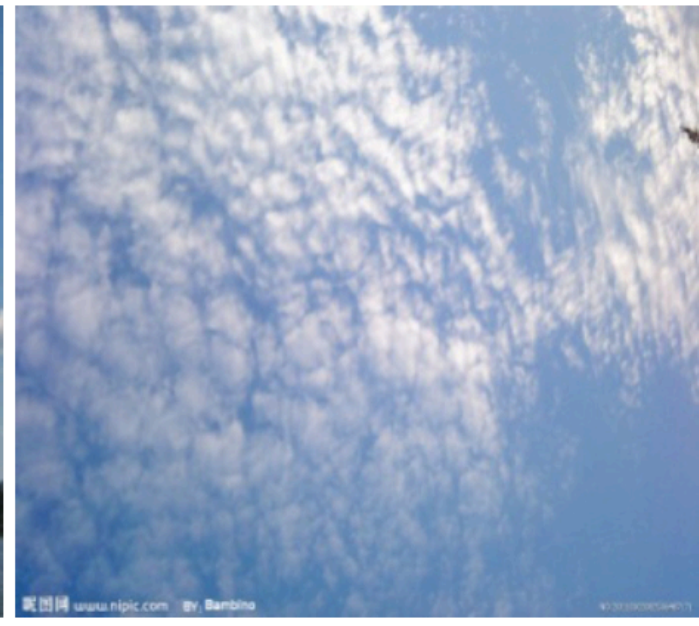

(b)

Fig. (5). Cumulus are mistaken for cirrus (a) and stratus (b). 


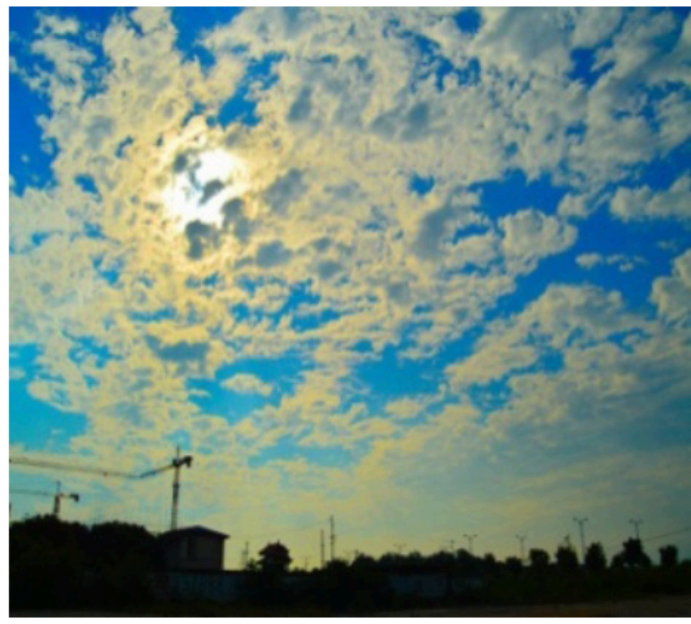

(a)

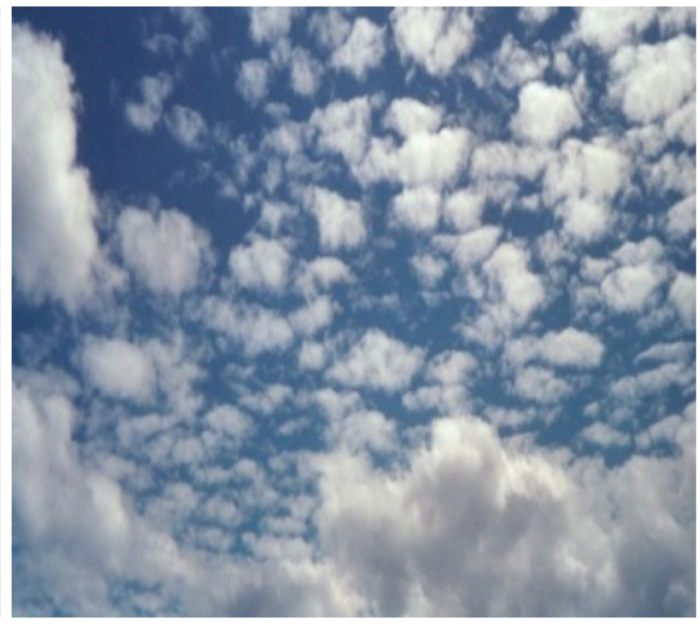

(b)

Fig. (6). Stratus are mistaken for cirrus (a) and cumulus (b).

check if these blocks have enough information for assigning the image blocks to a cloud type. It can be ensured that through the above suggestions, an improved algorithm is possible. In addition, other, features that were not mentioned, such as LBP features and shape features, may also improve the algorithm's performance. This work, however, only discussed the single type of sky cloud images, while the sky types are often complex. Therefore, future research is needed for automatic identification of complex cloud image.

\section{CONFLICT OF INTEREST}

The authors confirm that this article content has no conflict of interest.

\section{ACKNOWLEDGEMENTS}

This work was financially supported by the Six Talent Peaks Program of Jiangsu Province (2014-XXRJ-007), Natural Science Foundation of Jiangsu Province (BK20131002).

\section{REFERENCES}

[1] K.S. Carslaw, R.G. Harrison, and J. Kirkby, "Cosmic rays, clouds, and climate", Science, vol. 298, pp. 1732-1737, 2002.

[2] G.L. Stephens, "Cloud feedbacks in the climate system: A critical review", Journal of Climate, vol. 18, pp. 237-273, 2005.

[3] S. Solomon, D. Qin, M. Manning, Z. Chen, M. Marquis, K. Averyt, M. Tignor, and H. L. Miller, "Climate Change 2007: The Physical Science Basis - Contribution of Working group I to the $4^{\text {th }}$ Assessment Report of the IPCC", Newyork: Cambridge University Press, 2007, p. 996.

[4] J. Vazquez-Cuervo, E.M. Armstrong, and A. Harris, "The effect of aerosols and clouds on the retrieval of infrared sea surface temperatures", Journal of Climate, vol. 17, pp. 3921-3933, 2004.

[5] J E. Shields, R W. Johnson, and M E. Karr, "Automated day/night whole sky imagers for field assessment of cloud cover distributions and radiance distributions", $10^{\text {th }}$ Symposium on Meteorological Observations and Instrumentation, American Meteorological Society: Arizona, 1998, p. 401.

[6] C N. Long, and J. J. DeLuisi, "Development of an automated hemispheric sky imager for cloud fraction retrievals", In: Proceedings of $10^{\text {th }}$ Symposium on Meteorological Observations and Instrumentation, American Meteorological Society: Arizona, 1998.

[7] X. Sun, and J. Liu, "Correction of the non-uniformity of the radiometric detector of WSICMS", Proceedings of SPIE, China: SPIE. Digital Library, 2009.

[8] J. Yang, W. Lu, Y. Ma, and W. Yao, "An automated cirrus cloud detection method for a ground-based cloud image", Journal of Atmospheric Oceanic Technology, vol. 29, pp. 527-537, 2012

[9] J K. Buch, and C H. Sun, "Cloud classification using whole-sky imager data", The $9^{\text {th }}$ Symposium on Meteorological Observations and Instrumentation, Charlotte, North Carolina: AMS, pp. 353358, 1995.

[10] Peura, and P. Kostamo, "A new approach to land-based cloud classification", In: The Proceedings of the $13^{\text {th }}$ International Conference on Pattern Recognition, 1996.

[11] M. Singh, and M. Glennen, "Automated ground-based cloud recognition", Pattern Analysis Applications, vol. 8, pp. 258-271, 2005 .

[12] J. Calb'o, and J. Sabburg, "Feature extraction from wholeskyground-based images for cloud-type recognition", Journal of Atmospheric Ocean. Technology, vol. 25, pp. 3-14, 2008.

[13] A. Heinle, A. Macke, and A. Srivastav, "Automatic cloud classification of whole sky images," Atmospheric Measurement Technology, vol. 3, pp. 557-567, 2010.

[14] A. Kazantzidis and P. Tzoumanikas, "Cloud detection and classification with the use of whole-sky ground-based images". Atmospheric Research, vol. 113, pp. 80-88, 2012.

[15] K. Tuceryan, Texture Analysis, Handbook Pattern Recognition and Computer Vision, World Scientific: Singapore, 1993.

[16] R.M. Haralick, "Statistical and structural approaches to texture", Proceedings of the IEEE, vol. 67, no. 5, 1979 .

[17] H. Tamura, S. Mori, and T. Yamawaki, "Texture Fearures Corresponding to Visual Perception", "IEEE Transactions on Systems, Man and Cyberneties", vol. 8, pp. 460-473, 1978.

[18] M. Stricker, and M. Orengo, "Similarity of color images", SPIE Storage and Retrieval for Image and Vedio Databases, vol.2185, pp. 381-392, 1995.

[19] K. Grauman, and T. Darrell, "Pyramid match-ing kernel: Discriminative classification with sets of image features", Proceedings of the IEEE International Conference on Computer Vision, China: IEEE Xplore, 2005.

[20] J. Zhang, M. Marszalek, S. Lazebnik, and C. Schmid, "Local features and kernels for classification of texture and object categories", International Journal of Computer Vision, vol. 73, pp. 213-238, 2007.

[21] J. Winn, A. Criminisi, and T. Minka, "Object categorization by learned universal visual dictionary", Proceedings of the IEEE International Conference on Computer Vision, pp. 1800-1801, 2005. 
[22] G. Qiu, "Indexing chromatic and achromatic patterns for contentbased color image retrieval", Pattern Recognition, vol. 35, pp. 1675-1686, 2002.

[23] L. Feifei, "A bayesian hierarchical model for learning natural scene categories", Processing of IEEE Computer Society Conference on Computer Vision and Pattern Recognition, pp. 524-531, 2005.

[24] E. Nowak, F. Jurie, and B. Trigg, "Sampling strategies for bag-offeatures image classification", $E C C V$, pp. 490-503, 2006.
[25] J. Hartigan, and M. A. Wang, "K-means clustering algorithm", Applied Statistics, vol. 28, pp. 100-108, 1979.

[26] G. B. Huang. "Learning capability and storage capacity of twohidden-layer feed forward networks", IEEE Transactions on Neural Networks, vol. 14, pp. 274-281, 2003.

[27] G.B. Huang, Q.Y. Zhu, and C.K. Siew, "Extreme learning machine: Theory and application", Neurocomputing, vol. 70, pp. 489-501, 2006.

Received: June 10, 2015

Revised: July 29, 2015

Accepted: August 15, 2015

(C) Wu et al.; Licensee Bentham Open.

This is an open access article licensed under the terms of the (https://creativecommons.org/licenses/by/4.0/legalcode), which permits unrestricted, noncommercial use, distribution and reproduction in any medium, provided the work is properly cited. 